\title{
Urine opiate screening: false-positive result with levofloxacin
}

\author{
Qaiser Shafiq MD, Anand Mutgi MD
}

Previously published at www.cmaj.ca

A 96-year-old woman with moderate to severe dementia was admitted in acute delirium. According to her next of kin, the patient had been reporting generalized weakness for the last few days, and her appetite had decreased. The day before admission, she began having visual and auditory hallucinations.

At the time of admission, the patient was delirious and in moderate respiratory distress, with rapid shallow breathing and an oxygen saturation level of $88 \%$ on room air. Bilateral basilar crackles on inspiration and right-sided rhonchi were heard on auscultation. Her blood pressure was 140/75 mm Hg, her heart rate was 110 beats/min with a normal sinus rhythm, and her temperature was $37.2^{\circ} \mathrm{C}$. The initial results of imaging and laboratory investigation were all normal, including a computed tomography scan of the head, chest radiographs, complete blood count with differential count, thyroid function tests, and serum levels of electrolytes, liver enzymes, amylase, lipase, cardiac enzymes, vitamin $\mathrm{B}_{12}$, erythrocyte folate, iron, ammonia and alcohol. The results of her blood gas tests were normal except for a partial pressure of oxygen of 60 . The results of Gram staining and culture of blood, urine and sputum samples were negative for bacteria. The results of urine toxicology screening were negative for opiates and benzodiazepines. Twelve-lead electrocardiography did not show evidence of acute coronary syndrome or arrhythmias. Empirical treatment with broad-spectrum antibiotics, including piperacillintazobactam and vancomycin, was started for suspected aspiration pneumonia.

Three days later, the patient's mental status and respiratory distress were worse. Levofloxacin was added to the treatment to provide coverage for atypical bacteria. A neurology consultation was obtained to rule out a neurologic cause for the patient's worsening mental status. Magnetic resonance imaging of the brain and electroencephalography were done, but neither showed acute neurologic pathology. We diagnosed metabolic encephalopathy secondary to underlying pneumonia.

According to social services, the patient owned a home where she and a relative had been living together for several years. The patient had been receiving nursing care and other services in her home. Home health care staff had suspected physical abuse of the patient, and as a result, the court had appointed a lawyer as the patient's legal guardian. Given the change in the patient's mental status and upon learning about

\section{Key points}

- Commonly used medications can interact with enzyme immunoassay urine screening tests and result in falsepositive results for opiates and other substances.

- A complete review of the patient's medications should be performed while interpreting the results of a urine drug screen in the clinic.

- When the result of a urine drug screen is suspected of being falsely positive for opiates, confirmatory testing with a nonimmunologic, quantitative technique such as mass spectroscopy should be performed.

the alleged physical abuse, we were concerned about possible foul play and repeated the urine toxicology screen. To our surprise, the results were positive for opiates. A system-wide investigation was undertaken to find the possible source of the opiates. No evidence was found that an opiate-containing medication had been administered to the patient since the time of admission, and the nursing staff ruled out medication error.

While searching the literature for information on possible false-positive urine screens for opiates, we found an article by Baden and colleagues, ${ }^{1}$ which reviewed the association between such false-positive results and fluoroquinolones, such as levofloxacin, when using enzyme immunoassay techniques. Our laboratory uses the SYNCHRON enzyme immunoassay for urine toxicology screening, with a laboratory cutoff level for opiates of $300 \mathrm{ng} / \mathrm{mL}(1050 \mathrm{nmol} / \mathrm{L})$.

To confirm whether the urine screen result was false positive, the same urine sample that had tested positive for opiates and a serum sample taken within an hour of the urine sample were sent to an outlying facility for a nonimmunologic (mass spectroscopy) confirmatory test. Both samples tested negative for opiates with laboratory cutoff levels of $5 \mathrm{ng} / \mathrm{mL}$ $(17.5 \mathrm{nmol} / \mathrm{L})$ for urine and $2 \mathrm{ng} / \mathrm{mL}(7 \mathrm{nmol} / \mathrm{L})$ for serum.

We stopped treatment with levofloxacin and substituted it

From the Division of Endocrinology, Diabetes and Hypertension (Shafiq), Brigham and Women's Hospital, Harvard Medical School, Boston, USA; and the Department of Internal Medicine (Mutgi), University of Toledo Medical Center, Toledo, USA

CMAJ 2010. DOI:10.1503/cmaj.091508 
with azithromycin. One week later, the results of a repeat urine toxicology screen using the SYNCHRON enzyme immunoassay technique were negative for opiates. The patient's symptoms gradually improved, and she was discharged to an extended care nursing facility.

\section{Discussion}

Enzyme immunoassay qualitative techniques for urine screening are commonly used because of their wide availability, lower cost and reduced testing time compared with nonimmunologic techniques. Floroquinolones, such as levofloxacin, are frequently prescribed for the treatment of both community- and hospital-acquired infections. When enzyme immunoassay techniques are used for urine screening, a falsepositive result for opiates in a patient taking levofloxacin may lead to erroneous clinical decisions and inadvertent litigation. ${ }^{1-4}$ Baden and colleagues found that even a single dose of levofloxacin can interact with the enzyme immunoassay screening test, and the false-positive result for opiates can remain positive for 24 hours or more depending on the clearance of levofloxacin from the system. In their study involving six healthy volunteers, the researchers tested the crossreactivity of 13 different quinolones with five commercial opiate screening assays. Nine of the quinolones caused a false-positive result in at least one of the assays. Levofloxacin, ofloxacin and pefloxacin most consistently caused false-positive results.

Fluoroquinolones are not the only medications that may cross-react with enzyme immunoassay urine screening tests. Others include commonly used over-the-counter medications such as ibuprofen and dextromethorphan (Table 1). ${ }^{5}$ Venlafaxine, selegiline, oxaprozin and rifampin also have the potential to interact with these tests. Depending on the interaction, the test result may be false positive for benzodiazepines, opiates, amphetamines, barbiturates, cannabinoids or phencyclidine (PCP).

Table 1: Cross-reactivity of commonly used medications in urine toxicology screening assays

\begin{tabular}{|c|c|}
\hline $\begin{array}{l}\text { Drug identified in } \\
\text { false-positive result }\end{array}$ & $\begin{array}{l}\text { Medication } \\
\text { responsible for result }\end{array}$ \\
\hline $\begin{array}{l}\text { Amphetamine and } \\
\text { methamphetamine }\end{array}$ & $\begin{array}{l}\text { Selegiline, camphor/menthol vapour } \\
\text { inhaler }\end{array}$ \\
\hline Barbiturate & $\begin{array}{l}\text { Nonsteroidal anti-inflammatory } \\
\text { drugs (ibuprofen, naproxen) }\end{array}$ \\
\hline Benzodiazepine & Oxaprozin \\
\hline Cannabinoid & $\begin{array}{l}\text { Nonsteroidal anti-inflammatory } \\
\text { drugs (ibuprofen, naproxen) }\end{array}$ \\
\hline Opiate & $\begin{array}{l}\text { Fluoroquinolones (e.g., levofloxacin, } \\
\text { ofloxacin and pefloxacin), rifampin }\end{array}$ \\
\hline Phencyclidine & Venlafaxine, dextromethorphan \\
\hline
\end{tabular}

Source: Vincent et al.

If a false-positive urine screen result is suspected, a quantitative test using nonimmunologic techniques, such as gas chromatography, mass spectroscopy or high-performance liquid chromatography, is necessary for confirmation.

This article has been peer reviewed.

Competing interests: None declared.

\section{REFERENCES}

1. Baden LR, Horowitz G, Jacoby H, et al. Quinolones and false-positive urine screening for opiates by immunoassay technology. JAMA 2001;286:3115-9.

2. Straley CM, Cecil EJ, Herriman MP. Gatifloxacin interference with opiate urine drug screen. Pharmacotherapy 2006;26:435-9.

3. Zacher JL, Givone DM. False-positive urine opiate screening associated with fluoroquinolone use. Ann Pharmacother 2004;38:1525-8.

4. Meatherall R, Dai J. False-positive EMIT II opiates from ofloxacin. Ther Drug Monit 1997;19:98-9.

5. Vincent EC, Zebelman A, Goodwin C, et al. Clinical inquiries. What common substances can cause false positives on urine screens for drugs of abuse? J Fam Pract 2006;55:893-4, 897

Correspondence to: Dr. Qaiser Shafiq, Department of Internal Medicine, University of Toledo Medical Center, 3045 Arlington Ave., ToledoOH 43614,USA; qaiser.shafiq@utoledo.edu

\section{Watch for our first special digital issue}

CMAJ...

Selections in depression

Coming in

November

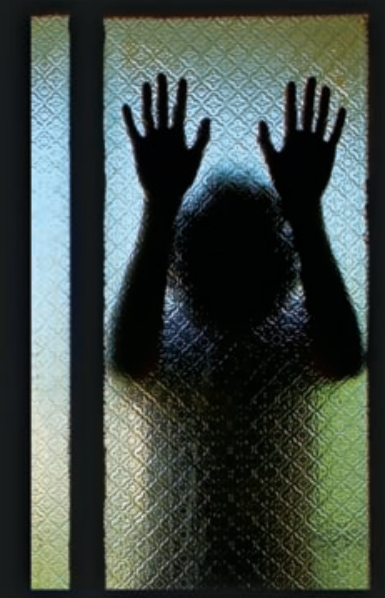

If you would like to receive this issue on depression, please email subscriptions@cma.ca

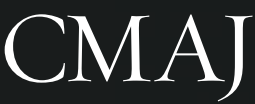

Medical knowledge that matters 